\title{
2-Uninormlar Üzerinde Denklik Bağıntısı ve Kıyaslanamayan Elemanların Kümesi
}

\author{
Equivalence Relation on 2-Uninorms and the Set of Incomparable Elements
}

\section{Ümit ERTUĞRUL*}

Karadeniz Teknik Üniversitesi, Fen Fakültesi Matematik Bölümü, 61080, Trabzon

\author{
• Geliş tarihi / Received: 14.12.2017 • Düzeltilerek geliş tarihi / Received in revised form: 02.04.2018 • Kabul tarihi / Accepted:04.04.2018
}

\section{$\ddot{O} z$}

Uninormlar, üçgensel normları, üçgensel konormları kapsayan birleştirme fonksiyonlarının bir sınıfıdır. 2-Uninormlar ise uninormları ve nullnormları kapsayan çok daha genel bir sınıftır. Uninormlardan elde edilen kısmen sıralama bağıntısı ve özellikleri üzerine yapılan çalışmalar, onların daha genel bir sınıfı olan 2-uninormlar için de bu tip özelliklerin araştırılmasını oldukça doğal kılar. Bu çalışmada, 2-uninormlardan elde edilen sıralama bağıntısı göz önüne alınarak, 2-uninormların sınıfı üzerinde bir denklik bağıntısı tanımlanmış ve bazı özellikleri araştırılmıştır. İlaveten, 2 uninormlardan elde edilen sıralama bağıntısına göre kıyaslanamayan elemanların kümesi karakterize edilmiştir.

Anahtar kelimeler: 2-Uninorm, Uninorm, Kısmen Sıralama Bağıntısı, Sınırlı Kafes

\begin{abstract}
Uninorms are a class of aggregation functions involving triangular norms and triangular conorms. 2-Uninorms are a much more general class that includes uninorms and nullnorms. Studies on partial order obtained from uninorms and their properties make it very natural to investigate such properties for their more general class 2-uninorms. In this study, an equivalence relation is defined on the class of 2-uninorms and some properties are investigated, taking into account the order relation obtained from 2-uninorms. In addition, the set of incomparable elements according to the ordering relation obtained from 2-uninorms is characterized.
\end{abstract}

Keywords: 2-Uninorm, Uninorm, Partial Order, Bounded Lattice

* Ümit ERTUĞRUL; uertugrul@ktu.edu.tr; Tel: (0462) 37725 76; orcid.org/0000-0003-0672-8134 


\section{Giriş}

Uninormlar ilk olarak [0,1] birim reel aralık üzerinde tanımlanmıştır (Yager vd., 2008). Uninorların, üçgensel normları ve üçgensel konormların daha genel bir sinıfi olması nedeniyle, araştırmacılar tarafından çok çalışılan bir konu olmuştur (Karaçal vd., 2017; Karaçal vd. 2015).

Üçgensel normlardan elde edilen kısmen sıralama bağıntısının tanımının verilmesinin (Karaçal vd., 2011) ve ardindan bu konuyu detaylı inceleyen bir çok çalışmanın yapılmasının ardından (Kesicioğlu vd. 2015; Kesicioğlu vd. 2014), bu tip bir siralama bağıntısına dair ilk girişim Hliněná, Kalina ve Král tarafindan yapılmıştır (Hliněná vd., 2014), fakat bu bağıntı bir kısmen sıralama bağıntıs1 değildir. Bu çalışmanın ardından, uninormlar üzerinde kısmen sıralama bağıntısı Ertuğrul, Kesicioğlu ve Karaçal tarafından tanımlanmıştır (Ertuğrul vd., 2016) ve çalışılmıştır (Kesicioğlu vd., 2017).

2-Uninormlar ilk olarak Akella tarafindan tanımlanmıştır (Akella, 2007) ve ardından bazı araştırmacılar tarafindan çalışılmıştır (Ertuğrul, 2017b). 2-Uninormların, uninormları dolayısıyla üçgensel normları ve üçgensel konormları kapsayan bir sinif olmasi yapilan sira çalışmalarının 2-uninormlar için de araştırılmasını doğal ve gerekli kılmıştır, bu bağlamda sınırlı kafesler üzerinde 2-uninormlardan elde edilen kısmen sıralama bağıntısı tanımlanmış ve bazı özellikleri incelenmiştir (Ertuğrul vd., 2017; Ertuğrul, 2017a).

$\mathrm{Bu}$ çalışma da ise, 2-uninormlardan elde edilen sıralama bağıntısı göz önüne alınarak, bir denklik bağıntısı tanımlanmıştır ve bu bağıntının bazı özellikleri incelenmiştir. İlaveten, 2uninormlardan elde edilen kısmen siralama bağıntısına göre kıyaslanamayan elemanların kümesi $K_{U^{2}}$ karakterize edilmiş ve yine 2uninormlardan elde edilen kismen siralama bağıntısına göre bir $x$ elemanı ile kıyaslanamayan tüm elemanların kümesi $I_{U^{2}}^{(x)}$ ile ilişkisi ortaya konulmuştur.

$\mathrm{Bu}$ çalışma üç ana bölümden oluşmaktadır. İkinci bölümde, üçüncü ve dördüncü bölümde kullanılacak veya buradaki çalışmalara temel teşkil edecek tanım, teorem ve önermelere yer verilmiştir. Üçüncü bölümde, 2-uninormlardan elde edilen sıralama bağıntısı göz önüne alınarak bir bağıntı tanımlanmış, bu bağıntının bir denklik bağıntıs1 olduğu gösterilmiş, 2-uninormun belirlediği üçgensel normlar ve konormlardan elde edilen siralama ile 2-uninormlardan elde edilen sıralama arasındanki ilişki araştırılmıştır. Dördüncü bölümde, 2-uninormlardan elde edilen sıralama bağıntısına göre kıyaslanamayan tüm elemanların kümesi $K_{U^{2}}$ nin karakterize edilmesinin yanı sira, $x$ elemanı ile kıyaslanamayan tüm elemanların kümesi $I_{U^{2}}^{(x)}$ ile ilişkisi de ortaya konmuştur.

\section{2. İlgili Tanım ve Teoremler}

Bu bölümde sonraki bölümlerde kullanılacak ilgili tanım, teorem ve önermelere yer verilmiştir.

2.1. Tanım (Birkhoff, 1967) $P$ bir küme ve $\leq, P$ üzerinde bir bağıntı olsun. Her $x, y, z \in P$ için
P1. Her $x \in P$ için $x \leq x$
(Yansima)

P2. $x, y \in P$ için $x \leq y$ ve $y \leq x$ ise $x=y$

(Ters Simetri)

P3. $x, y, z \in P$ için $x \leq y$ ve $y \leq z$ ise $x \leq z$

(Geçişme)

şartları sağlanırsa, $\leq$ bağıntısına $P$ üzerinde bir siralama (veya kısmen sıralama) denir. Üzerinde bir $\leq$ sıralama bağıntısı mevcut olan $P$ kümesine sıralı küme (veya kısmen sıralı küme) denir ve bu küme $(P, \leq)$ ikilisi ile gösterilir.

2.2 Tanım (Karaçal vd., 2011) $(P, \leq)$ kısmen siralı bir küme olsun. $a, b \in P$ için $a \nsubseteq b$ ve $b \$ a$ ise yani $a$ ve $b$ elemanları kıyaslanmıyorsa $a$ ve $b$ elemanlarına kiyaslanamayan elemanlar denir ve bu $a \| b$ ile gösterilir. $c \in P$ için $c$ elemanı ile kıyaslanamayan elemanların kümesi

$I_{c}:=\{x \in P: x \| c\}$

ile gösterilir.

2.3. Tanım (Birkhoff, 1967) $(L, \leq)$ bir k1smen siralı kümesi olsun. Her $x, y \in L$ için $\sup \{x, y\}$ ve $\inf \{x, y\}$ mevcut ise $L$ ye kafes denir. $\square$

2.4. Tanım (Birkhoff, 1967) Bir $L$ kafesine sinırlı kafes denir: $\Leftrightarrow L$, en küçük eleman 0 ve en büyük eleman 1 e sahiptir. Bu durum, kısaca $(L, \leq, 0,1)$ ile gösterilir. $\square$

2.5. Tanım (Karaçal vd., 2011) $L$ sınırlı bir kafes olsun. Bir üçgensel norm $T$ (kısaca t-norm) $L$ üzerinde komütatiflik, birleşme, monotonluk özelliklerini sağlayan 1- birim elemanlı bir ikili işlemdir. ᄆ 
2.6. Tanım (Karaçal vd., 2011) $L$ sınırlı bir kafes olsun. Bir üçgensel konorm S (kısaca t-konorm) $L$ üzerinde komütatif, birleşme, monoton özelliklerini sağlayan 0- birim elemanlı bir ikili işlemdir. ᄆ

2.7. Tanım (Karaçal vd., 2011) Sınırlı bir $L$ kafesi üzerindeki bir t-norm $T$ ye bölünebilirdir denir : $\Leftrightarrow x \leq y$ olan her $x, y \in L$ için $x=$ $T(y, z)$ olacak şekilde bir $z \in L$ mevcuttur. $\square$

2.8. Önerme (Karaçal vd., 2011) $T, L=[0,1]$ üzerinde bir t-norm olsun. $T$ bölünebilirdir $\Leftrightarrow T$ süreklidir. $\square$

2.9. Tanım (Ertuğrul, 2018) Bir nullnorm $(L, \leq, 0,1)$ sınırlı kafesi üzerinde aşağıdaki özellikleri sağlayan bir fonksiyondur; yani $V: L^{2} \rightarrow L$ fonksiyonuna bir nullnorm denir : $\Leftrightarrow$ Her $x, y, z \in L$ için

V1. $V(x, y)=V(y, x)$

(Komütatiflik)

V2. $V(x, V(y, z))=V(V(x, y), z) \quad$ (Birleşme)

V3. $y \leq z$ ise $V(x, y) \leq V(x, z) \quad$ (Monotonluk)

V4. $x \leq a$ ise $V(x, 0)=x$ ve $x \geq a$ ise $V(x, 1)=$ $x$ olacak şekilde bir $a \in L$ mevcuttur. $\square$

2.10. Tanım (Grabish vd., 2009) $(L, \leq, 0,1)$ sinırl bir kafes olsun. Bir uninorm $U: L^{2} \rightarrow L$, komütatiflik, birleşme ve monotonluk özelliklerini sağlayan, $e \in L$ birim elemanlı ( $L$ nin her $x$ elemanı için $U(e, x)=x)$ bir ikili işlemdir. $\square$

2.11. Önerme (Grabish vd., 2009) $(L, \leq, 0,1)$ sınırlı bir kafes ve $U, L$ üzerinde $e \in L$ birimli bir uninorm olsun. $\mathrm{O}$ halde,

(i) $T_{U}=U \downarrow[0, e]^{2}:[0, e]^{2} \rightarrow[0, e], \quad[0, e]$ üzerinde bir t-normdur.

(ii) $S_{U}=U \downarrow[e, 1]^{2}:[e, 1]^{2} \rightarrow[e, 1], \quad[e, 1]$ üzerinde bir t-konormdur. $\square$

2.12. Tanım (Grabish vd., 2009) $(L, \leq, 0,1)$ sinırlı bir kafes ve $U, L$ üzerinde bir uninorm olsun. $U(0,1)=0$ ise $U$ uninormuna konjanktif uninorm, $U(0,1)=1$ ise $U$ uninormuna disjanktif uninorm denir. $\square$

2.13. Tanım (Akella, 2007) $(L, \leq, 0,1)$ sinırlı bir kafes olsun. Bir 2-uninorm $U^{2}: L^{2} \rightarrow L$ komütatiflik, birleşme, monotonluk özelliklerini sağlayan ve $0 \leq e \leq k \leq f \leq 1$ şartını sağlayan $e, k, f \in L$ için

$x \leq k$ olan her $x \in L$ için $U^{2}(e, x)=x$ ve $x$

$$
\geq k \text { olan her } x \in L \text { için } U^{2}(f, x)
$$$$
=x
$$

özelliklerini sağlayan bir ikili işlemdir. Tüm 2uninormların sınıfı $U_{k(e, f)}$ ile gösterilecektir. $\square$

2.14. Önerme (Ertuğrul, 2017b) $(L, \leq, 0,1)$ sinırlı bir kafes ve $U^{2} \in U_{k(e, f)}$ olsun. Bu takdirde, $\quad U^{2} \downarrow_{[0, k]^{2}}=U_{1}^{2} \quad$ ve $\quad U^{2} \downarrow_{[k, 1]^{2}}=U_{2}^{2}$ olarak gösterilirse, $U_{1}^{2}[0, k]$ üzerinde $e$ birim elemanl1 bir uninorm ve $U_{2}^{2}[k, 1]$ üzerinde $f$ birim elemanlı bir uninormdur. $\square$

2.15. Önerme (Ertuğrul, 2017b) $(L, \leq, 0,1)$ sinırlı bir kafes, $U^{2} \in U_{k(e, f)}$ ve $U^{2} \downarrow_{[0, k]^{2}}=U_{1}^{2}$ ve $U^{2} \downarrow_{[k, 1]^{2}}=U_{2}^{2}$ ile gösterilsin. Bu takdirde $U_{1}^{2} \downarrow_{[0, e]^{2}}=T_{U_{1}^{2}}[0, e]$ üzerinde $e$ birim elemanlı bir t-norm, $U_{1}^{2} \downarrow_{[e, k]^{2}}=S_{U_{1}^{2}}[e, k]$ üzerinde $e$ birim elemanlı bir t-konorm, $U_{2}^{2} \downarrow_{[k, f]^{2}}=T_{U_{2}^{2}}$ $[k, f]$ üzerinde $f$ birim elemanlı bir t-norm ve $U_{2}^{2} \downarrow_{[f, 1]^{2}}=S_{U_{2}^{2}}[f, 1]$ üzerinde $f$ birim elemanlı bir t-konormdur.

2.16. Teorem (Ertuğrul, 2017b) $(L, \leq, 0,1)$ sinırlı bir kafes, $U_{1}[0, k]$ üzerinde $e$ birim elemanlı disjanktif uninorm ve $U_{2}[k, 1]$ üzerinde $f$ birim elemanlı konjanktif uninorm olsun. $\mathrm{Bu}$ takdirde,

$U^{2}(x, y)=\left\{\begin{array}{cc}U_{1}(x, y), & (x, y) \in[0, k]^{2}, \\ U_{2}(x, y), & (x, y) \in[k, 1]^{2}, \\ k, & \text { aksi halde }\end{array}\right.$

ile tanımlı operatör $U_{k(e, f)}$ nin bir elemanıdır, yani bir 2-uninormdur. $\square$

2.17. Tanım (Karaçal vd., 2011) $L$ sınırlı bir kafes ve $T, L$ üzerinde bir t-norm olsun. T-norm $T$ için $T$-kısmen sıra (üçgensel sıra) aşağıdaki gibi tanımlanır ve $\leq_{T}$ ile gösterilir: $x \leq_{T} y: \Leftrightarrow$ $T(\ell, y)=x$ olacak şekilde bir $\ell \in L$ elemanı mevcuttur. Dual olarak $S$-kısmen tanımlanır. 口

2.18. Tanım (Ertuğrul vd., 2016) $(L, \leq, 0,1)$ sinırlı bir kafes ve $U, e$ birim elemanlı bir uninorm olsun. $x, y \in L$ için aşağıdaki bağıntıyı tanımlayalım: 


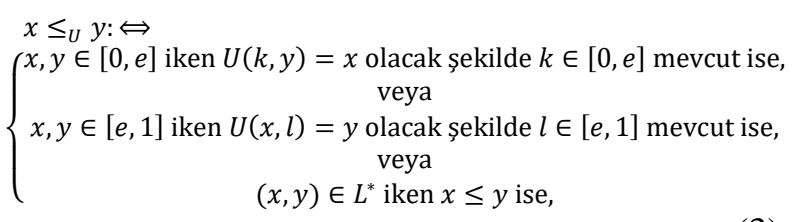

burada $L^{*}=[0, e] \times[e, 1] \cup[0, e] \times I_{e} \cup[e, 1] \times$ $[0, e] \cup[e, 1] \times I_{e} \cup I_{e} \times[0, e] \cup I_{e} \times[e, 1] \cup$ $I_{e} \times I_{e}$ dir. $\square$

2.19. Önerme (Ertuğrul vd., 2016) (3) ile tanımlanan bağıntı $L$ üzerinde bir kısmen sıralama bağıntısıdır. $\square$

2.20. Önerme (Ertuğrul vd., 2016) $(L, \leq, 0,1)$ sinırlı bir kafes ve $U, L$ üzerinde $e$ birim elemanlı bir uninorm olsun. $T_{U}$ ve $S_{U}$ bölünebilirdir ancak ve ancak $\leq_{U}=\leq$.

2.21. Tanım (Ertuğrul vd., 2017) $(L, \leq, 0,1)$ sınırlı bir kafes üzere $U^{2} \in U_{k(e, f)}$ olsun. $x, y \in L$ için

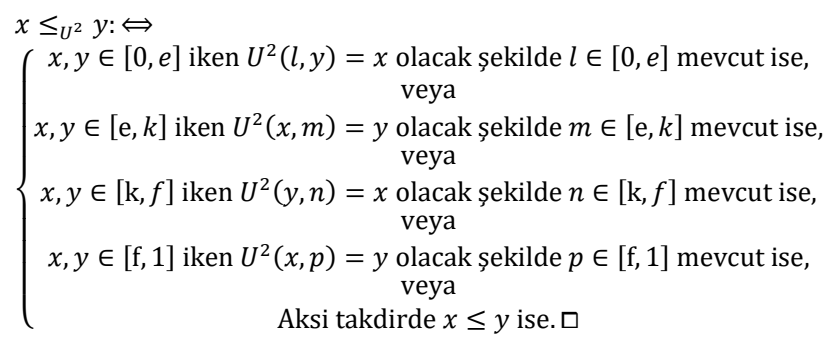

2.22. Önerme (Ertuğrul vd., 2017) (4) ile tanımlanan bağıntı $L$ üzerinde bir kısmen sıralama bağıntısıdır. $\square$

2.23. Önerme (Ertuğrul vd., 2017) $(L, \leq, 0,1)$ sinırlı bir kafes ve $U^{2} \in U_{k(e, f)}$ olsun. $x, y \in L$ için $x \leq_{U^{2}} \mathrm{y}$ ise, $x \leq y$ dir.

2-uninormlar uninormları kapsayan daha genel sınıflardır, bu sebeple uninormlar için yapılan çalışmaların 2-uninormlar ve dahası n-uninormlar için araştırılması araştırmacıların ilgilendiği çalışma konularıdır. $\mathrm{Bu}$ çalışmanın bundan sonraki kısmında uninormlar için yapılan araştırmalar 2-uninormlar için de araştırılacaktır. Elde edilecek sonuçlar, uninormlar için elde edilen sonuçların daha geneli olmak üzere $U^{2} \in U_{k(e, f)}$ için $e$ ve $f$ birim elemanları $e=f$ koşulunu sağladığında bu sonuçlar uninormlar için elde edilen sonuçlarla çakışacaktır.

\section{2-Uninormlar Üzerinde Denklik Bağıntısı}

Üçgensel normlar (konormlar), uninormlar ve nullnormlar için denklik sınıflarının araştırılmış olduğu göz önüne alınınca bu sınıfları kapsayan 2uninormlar için de bu tip bir araştırmanın yapılıyor olması oldukça anlamlıdır. Bu bölümde, 2-uninormlar için bir tip denklik bağıntısı tanımlanacak, bu denklik bağıntısının bazı özellikleri araştırılacaktır.

3.1. Tanım $(L, \leq, 0,1)$ sinırlı bir kafes olsun. $U_{k(e, f)} \quad$ sinıfi üzerinde $U^{2(1)}, U^{2(2)} \in U_{k(e, f)}$ olmak üzere $\sim$ bağıntısı şu şekilde tanımlansın:

$$
U^{2(1)} \sim U^{2(2)}
$$

$\Leftrightarrow U^{2(1)}$ den elde edilen kısmen sıra ile $U^{2(2)}$ den elde edilen kısmen sıra çakışır.

3.2. Önerme 3.1. Tanım'da verilen bağıntısı, $U_{k(e, f)}$ sınıfı üzerinde bir denklik bağıntısıdır. $\square$

Ispat. $\quad U^{2}, U^{2(1)}, U^{2(2)}, U^{2(3)} \in U_{k(e, f)} \quad$ keyfi alınsin.

i. Her $U^{2} \in U_{k(e, f)}$ keyfi 2-uninormu için $U^{2} \sim U^{2}$ olduğu açıktır.

ii. $\quad U^{2(1)}, U^{2(2)} \in U_{k(e, f)}$ ve $\quad U^{2(1)} \sim U^{2(2)}$ olsun. Buradan $U^{2(1)}$ den elde edilen kısmen sira ve $U^{2(2)}$ den elde edilen kısmen sıra çakışır. Böylece açıkça, $U^{2(2)} \sim U^{2(1)}$ olduğu elde edilir.

iii. $\quad U^{2(1)}, U^{2(2)}, U^{2(3)} \in U_{k(e, f)}, U^{2(1)} \sim U^{2(2)}$ ve $U^{2(2)} \sim U^{2(3)}$ olsun. Buradan, $U^{2(1)}$ den elde edilen kismen sira ile $U^{2(2)}$ den elde edilen kismen sira ve $U^{2(2)}$ den elde edilen kismen sira ile $U^{2(3)}$ den elde edilen kısmen sıra çakışırlar. Böylece, $U^{2(1)}$ den elde edilen kismen sira ile $U^{2(3)}$ den elde edilen kısmen sıra çakışır ve buradan da $U^{2(1)} \sim U^{2(3)}$ olduğu elde edilir.

Böylece, $\sim$ bağıntısının, $U_{k(e, f)}$ sınıfı üzerinde bir denklik bağıntısı olduğu elde edilir. $\square$

3.3. Tanım $(L, \leq, 0,1)$ sinırlı bir kafes ve $U^{2} \in U_{k(e, f)}$ olsun. $U^{2} \in U_{k(e, f)}$ nin $\sim$ denklik bağıntısına göre denklik sınıfı

$\overline{U^{2}}=\left\{U^{2(*)}: U^{2(*)} \sim U^{2}\right\}$

ile verilir. $\square$ 
3.4 Önerme $(L, \leq, 0,1)$ sinırlı bir kafes ve $U^{2(1)}, U^{2(2)} \in U_{k(e, f)} \quad$ olsun. Eğer $T_{U_{1}^{2(1)}}, S_{U_{1}^{2(1)}}, T_{U_{2}^{2(1)}}, S_{U_{2}^{2(1)}}, \quad T_{U_{1}^{2(2)}}, S_{U_{1}^{2(2)}}, T_{U_{2}^{2(2)}}$ ve $S_{U_{2}^{2(2)}}$ bölünebilir ise $U^{2(1)} \sim U^{2(2)}$ dir.

Ispat. $T_{U_{1}^{2(1)}}, S_{U_{1}^{2(1)}}, T_{U_{2}^{2(1)}}, S_{U_{2}^{2(1)}}$, $T_{U_{1}^{2(2)}}, S_{U_{1}^{2(2)}}, T_{U_{2}^{2(2)}}$ ve $S_{U_{2}^{2(2)}}$ bölünebilir ise $\leq_{T U_{1}^{2(1)}}=\leq_{S_{U_{1}^{2(1)}}}=\leq_{T_{U_{2}^{2(1)}}}=\leq_{S_{U_{2}^{2(1)}}}=\leq_{T_{U_{1}^{2(2)}}}=$ $\leq_{U_{1}^{2(2)}}=\leq_{T_{U_{2}^{2(2)}}}=\leq_{S_{U_{2}^{2(2)}}}=\leq$ olduğunu verir. Buradan da, $\leq_{U^{2(1)}}=\leq_{U^{2(2)}}=\leq$ olduğu elde edilir. Böylece, $U^{2(1)} \sim U^{2(2)}$ olur. .

\subsection{Uyarı}

i) 3.4 Önerme'deki $L$ kafesi $[0,1]$ birim reel aralik alınır ve $T_{U_{1}^{2(1)}}, S_{U_{1}^{2(1)}}, T_{U_{2}^{2(1)}}, S_{U_{2}^{2(1)}}$, $T_{U_{1}^{2(2)}}, S_{U_{1}^{2(2)}}, T_{U_{2}^{2(2)}}$ ve $S_{U_{2}^{2(2)}}$ sürekli olacak şekilde $U^{2(1)}, U^{2(2)}, \in U_{k(e, f)}$ seçilirse 2.8 . Önerme ve 2.20 Önerme'lerden $\leq_{U^{2(1)}}=$ $\leq_{U^{2(2)}}=\leq$ olup $U^{2(1)} \sim U^{2(2)}$ elde edilir.

ii) 3.4. Önerme'nin tersi doğru olmak zorunda değildir. Örnek 3.6 buna bir örnek teşkil eder.

3.6. Örnek $T^{n M}(x, y),[0, e]$ üzerinde aşağıdaki gibi

$T^{n M}(x, y)=\left\{\begin{array}{cc}0, & x+y \leq e \\ \min (x, y), & \text { aksi halde }\end{array}\right.$

$T^{n M},[k, f]$ üzerinde aşağıdaki gibi

$T^{n M}(x, y)= \begin{cases}0, & x+y \leq f \\ \min (x, y), & \text { aksi halde }\end{cases}$

$S_{D} \mathrm{t}$-konormu $[e, k]$ üzerinde aşağıdaki gibi

$S_{D}(x, y)= \begin{cases}k, & (x, y) \in(e, k)^{2} \\ \operatorname{mak}(x, y), & \text { aksi halde }\end{cases}$

ve $S_{D}$ t-konormu, $[f, 1]$ üzerinde aşağıdaki gibi

$S_{D}(x, y)= \begin{cases}f, & (x, y) \in(f, 1)^{2} \\ \operatorname{mak}(x, y), & \text { aksi halde }\end{cases}$

seçilirse $U_{1}=U_{\min }\left(T^{n M}, S_{D}, e\right)[0, k]$ üzerinde bir disjanktif uninorm ve $U_{2}=U_{\min }\left(T^{n M}, S_{D}, f\right)$ $[k, 1]$ üzerinde bir konjanktif uninormdur (Beliakov vd., 2007). (2) ile $U^{2(1)}(x, y)=\left\{\begin{array}{cc}U_{1}(x, y), & (x, y) \in[0, k]^{2}, \\ U_{2}(x, y), & (x, y) \in[k, 1]^{2}, \\ k, & \text { aksi halde }\end{array}\right.$
$[0,1]$ üzerinde bir 2-uninormdur.

Benzer düşünceyle, $T^{*}(x, y),[0, e]$ üzerinde aşağıdaki gibi

$T^{*}(x, y)=\left\{\begin{array}{lc}\frac{e}{2}, & x, y=\frac{e}{2} \\ T^{n M}, & \text { aksi halde }\end{array}\right.$

$T^{n M},[k, f]$ üzerinde aşağıdaki gibi

$T^{n M}(x, y)= \begin{cases}0, & x+y \leq f \\ \min (x, y), & \text { aksi halde }\end{cases}$

$S_{D}$ t-konormu $[e, k]$ üzerinde aşağıdaki gibi

$S_{D}(x, y)= \begin{cases}k, & (x, y) \in(e, k)^{2} \\ \operatorname{mak}(x, y), & \text { aksi halde }\end{cases}$

ve $S_{D}$ t-konormu, $[f, 1]$ üzerinde aşağıdaki gibi

$S_{D}(x, y)= \begin{cases}f, & (x, y) \in(f, 1)^{2} \\ \operatorname{mak}(x, y), & \text { aksi halde }\end{cases}$

seçilirse $U_{3}=U_{\min }\left(T^{*}, S_{D}, e\right)[0, k]$ üzerinde bir disjanktif uninorm ve $U_{2}=U_{\min }\left(T^{n M}, S_{D}, f\right)$ $[k, 1]$ üzerinde bir konjanktif uninormdur (Beliakov vd., 2007). (2) ile

$U^{2(2)}(x, y)=\left\{\begin{array}{cr}U_{3}(x, y), & (x, y) \in[0, k]^{2} \\ U_{2}(x, y), & (x, y) \in[k, 1]^{2} \\ k, & \text { aksi halde }\end{array}\right.$

$[0,1]$ üzerinde bir 2-uninormdur. $U^{2(1)}$ ve $U^{2(2)} 2$ uninormları sadece $[0, e]^{2}$ üzerinde farkl111k gösterir. $U^{2(1)} \downarrow_{[0, e]^{2}}=T^{n M}$ ve $U^{2(2)} \downarrow_{[0, e]^{2}}=T^{*}$ olup $[0, e]^{2}$ üzerinde $\leq_{T^{n M}}=\leq_{T^{*}}$ olduğundan $\leq_{U^{2(1)}}=\leq_{U^{2(2)}}$ olduğu elde edilir. Fakat açıkça, $U^{2(1)}\left(\frac{e}{2}, \frac{e}{2}\right)=T^{n M}\left(\frac{e}{2}, \frac{e}{2}\right)=0 \neq \frac{e}{2}=T^{*}\left(\frac{e}{2}, \frac{e}{2}\right)=$ $U^{2(2)}\left(\frac{e}{2}, \frac{e}{2}\right)$ olduğundan $U^{2(1)} \neq U^{2(2)}$ dir. $\square$

3.7.Teorem $(L, \leq, 0,1)$ sinırlı bir kafes ve $U^{2(1)}, U^{2(2)} \in U_{k(e, f)} \quad$ olsun. $T_{U_{1}^{2(1)}} \sim T_{U_{1}^{2(2)}}, S_{U_{1}^{2(1)}} \sim S_{U_{1}^{2(2)}}, T_{U_{2}^{2(1)}} \sim T_{U_{2}^{2(2)}} \quad$ ve $S_{U_{2}^{2(1)}} \sim S_{U_{2}^{2(2)}}$ dir ancak ve ancak $U^{2(1)} \sim U^{2(2)}$ dir. $\square$

Ispat. $x, y \in L$ için $x \leq_{U^{2(1)}} y$ olsun.

- $x, y \in[0, e] \quad$ ise $\quad U^{2(1)} \downarrow_{[0, e]^{2}}=T_{U_{1}^{2(1)}}$ olduğundan $x \leq_{U^{2(1)}} y$ olmas1 $x \leq_{T_{U_{1}^{2(1)}}} y$ olduğunu verir. Buradan, $T_{U_{1}^{2(1)}} \sim T_{U_{1}^{2(2)}}$ olduğu için $\quad x \leq_{T_{U_{1}^{2(2)}}} y$ olduğu elde edilir. 
$U^{2(2)} \downarrow_{[0, e]^{2}}=T_{U_{1}^{2(2)}}$ olduğundan, $x \leq_{T_{U_{1}^{2(2)}}} y$ olmasi $x \leq_{U^{2(2)}} y$ olmasin1 gerektirir.

- $x, y \in[e, k] \quad$ ise $\quad U^{2(1)} \downarrow_{[e, k]^{2}}=S_{U_{1}^{2(1)}}$ olduğundan $x \leq_{U^{2(1)}} y$ olmasi $x \leq_{U_{U^{2(1)}}} y$ olduğunu verir. Buradan, $S_{U_{1}^{2(1)}} \sim S_{U_{1}^{2(2)}}$ olduğu için $\quad x \leq_{S_{U_{1}^{2(2)}}} y$ olduğu elde edilir. $U^{2(2)} \downarrow_{[e, k]^{2}}=S_{U_{1}^{2(2)}}$ olduğundan, $x \leq_{S_{U_{1}^{2(2)}}} y$ olmasi $x \leq_{U^{2(2)}} y$ olmasinı gerektirir.

- $x, y \in[k, f] \quad$ ise $U^{2(1)} \downarrow_{[k, f]^{2}}=T_{U_{2}^{2(1)}}$ olduğundan $x \leq_{U^{2(1)}} y$ olmas1 $x \leq_{T_{2}^{2(1)}} y$ olduğunu verir. Buradan, $T_{U_{2}^{2(1)}} \sim T_{U_{2}^{2(2)}}$ olduğu için $\quad x \leq_{T_{U_{2}^{2(2)}}} y$ olduğu elde edilir. $U^{2(2)} \downarrow_{[k, f]^{2}}=T_{U_{2}^{2(2)}}$ olduğundan, $x \leq_{T_{U_{2}^{2(2)}}} y$ olmasi $x \leq_{U^{2(2)}} y$ olmasinı gerektirir.

- $x, y \in[f, 1] \quad$ ise $\quad U^{2(1)} \downarrow_{[f, 1]^{2}}=S_{U_{2}^{2(1)}}$ olduğundan $x \leq_{U^{2(1)}} y$ olmasi $x \leq_{S_{2}^{2(1)}} y$ olduğunu verir. Buradan, $S_{U_{2}^{2(1)}} \sim S_{U_{2}^{2(2)}}$ olduğu için $\quad x \leq_{S_{U_{2}^{2(2)}}} y$ olduğu elde edilir. $U^{2(2)} \downarrow_{[f, 1]^{2}}=S_{U_{2}^{2(2)}}$ olduğundan, $x \leq_{S_{U_{2}^{2(2)}}} y$ olmas1 $x \leq_{U^{2(2)}} y$ olmasin1 gerektirir.

- Aksi takdirde, $x \leq_{U^{2(1)}} y$ olmasi $x \leq y$ olmasını, bu ise $x \leq_{U^{2(2)}} y$ olduğunu verir.

Benzer şekilde, $T_{U_{1}^{2(1)}} \sim T_{U_{1}^{2(2)}}, S_{U_{1}^{2(1)}} \sim S_{U_{1}^{2(2)}}, T_{U_{2}^{2(1)}} \sim T_{U_{2}^{2(2)}} \quad$ ve $S_{U_{2}^{2(1)}} \sim S_{U_{2}^{2(2)}}$ varsayımları altında $x \leq_{U^{2(2)}} y$ olması $x \leq_{V} y$ olduğunu verir. Böylece, $T_{U_{1}^{2(1)}} \sim T_{U_{1}^{2(2)}}, S_{U_{1}^{2(1)}} \sim S_{U_{1}^{2(2)}}, T_{U_{2}^{2(1)} \sim T_{U_{2}^{2(2)}}} \quad$ ve $S_{U_{2}^{2(1)}} \sim S_{U_{2}^{2(2)}}$ ise $U^{2(1)} \sim U^{2(2)}$ olduğu elde edilir.

Tersine, $U_{1}^{2} \sim U_{2}^{2}$ olsun. $\mathrm{O}$ halde, $\leq_{U^{2(1)}}=\leq_{U^{2(2)}}$

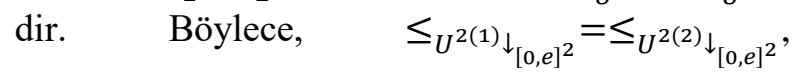

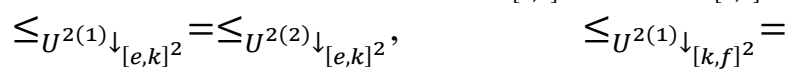
$\leq_{U^{2(2)} \downarrow_{[k, f]^{2}}}$ ve $\leq_{U^{2(1)} \downarrow_{[f, 1]^{2}}}=\leq_{U^{2(2) \downarrow_{[f, 1]^{2}}}}$ olduğu açıktır ve bu eşitliklerden sirasıyla $\leq_{T_{1}^{2(1)}}=$ $\leq_{T U_{1}^{2(2)}}, \quad \leq_{S_{U_{1}^{2(1)}}}=\leq_{S_{U_{1}^{2(2)}}}, \quad \leq_{T_{U_{2}^{2(1)}}}=\leq_{T_{U_{2}^{2(2)}}}$ ve $\leq_{U_{U_{2}^{2(1)}}}=\leq_{S_{U_{2}^{2(2)}}}$ olduğu elde edilir. $\mathrm{Bu}$ ise, $T_{U_{1}^{2(1)}} \sim T_{U_{1}^{2(2)}}, S_{U_{1}^{2(1)}} \sim S_{U_{1}^{2(2)}}, T_{U_{2}^{2(1)} \sim T_{U_{2}^{2(2)}} \quad \text { ve }}$ $S_{U_{2}^{2(1)}} \sim S_{U_{2}^{2(2)}}$ olduğunu verir. $\square$

\section{4. $\leq_{U^{2}}$ Siralamasına Göre Kıyaslanamayan Tüm Elemanların Kümesi $K_{U^{2}}$}

$\mathrm{Bu}$ bölümde, $(L, \leq, 0,1)$ sinırlı kafesi üzerinde tanımlı $U^{2} \in U_{k(e, f)}$ uninormu için $S_{U^{2}}$ siralamasına göre kiyaslanamayan elemanların kümesi $K_{U^{2}}$ kümesinin bazı özellikleri araştırılmış ve $\quad K_{U^{2}}=K_{T_{U_{1}^{2}}} \cup K_{S_{U_{1}^{2}}} \cup I_{e} \cup M_{e} \cup K_{T_{U_{2}^{2}}} \cup$ $K_{S_{U_{2}^{2}}} \cup I_{f} \cup M_{f} \cup I_{k}$ eşitliği ve $\leq_{U^{2}}$ siralamasına göre $x$ elemanı ile kıyaslanamayan elemanların kümesi $I_{U^{2}}^{(x)}$ ile $K_{U^{2}}$ nin ilişkisi ortaya koyulmuştur.

4.1. Tanım $(L, \leq, 0,1)$ sinırlı bir kafes ve $U^{2} \in U_{k(e, f)}$ olsun.

$$
\begin{aligned}
& K_{U^{2}}=\{x \in L \backslash\{0,1\} \\
& :\left(x<y \text { ve } x \$_{U^{2}} y\right) \text { veya }(y \\
& \left.<x \text { ve } y \$_{U^{2}} x\right) \text { veya } x \| y \text { olacak şekilde } y \\
& \in L \backslash\{0,1\} \text { mevcuttur. }\}
\end{aligned}
$$

$K_{T_{U_{1}^{2}}}=\{x \in[0, e] \backslash\{0, e\}$

: $\left(x<y\right.$ ve $\left.x \sharp_{T_{U_{1}^{2}}} y\right)$ veya $(y$

$<x$ ve $y{\bigsqcup_{T} U_{1}^{2}} x)$ veya $x \| y$ olacak şekilde $y$ $\in x \in[0, e] \backslash\{0, e\}$ mevcuttur. $\}$

$K_{S_{U_{1}^{2}}}=\{x \in[e, k] \backslash\{e, k\}$

: $\left(x<y\right.$ ve $\left.x \Varangle_{S_{U_{1}^{2}}} y\right)$ veya $(y$

$<x$ ve $\left.y \$_{S_{U_{1}^{2}}} x\right)$ veya $x \| y$ olacak şekilde $y$ $\in x \in[e, k] \backslash\{e, k\}$ mevcuttur. $\}$

$K_{T_{U_{2}^{2}}}=\{x \in[k, f] \backslash\{k, f\}$

: $\left(x<y\right.$ ve $\left.x \$_{T_{U_{2}^{2}}} y\right)$ veya $(y$

$<x$ ve $\left.y \Varangle_{T_{U_{2}^{2}}} x\right)$ veya $x \| y$ olacak şekilde $y$ $\in x \in[k, f] \backslash\{k, f\}$ mevcuttur. $\}$

$K_{S_{U_{2}^{2}}}=\{x \in[f, 1] \backslash\{f, 1\}$

: $\left(x<y\right.$ ve $\left.x \$_{S_{U_{2}^{2}}} y\right)$ veya $(y$

$<x$ ve $y{\$_{S_{U_{2}^{2}}}} x)$ veya $x \| y$ olacak şekilde $y$ $\in x \in[f, 1] \backslash\{f, 1\}$ mevcuttur. $\}$

olarak tanımlanır.

4.2. Önerme $(L, \leq, 0,1)$ sinırlı bir kafes ve $U^{2} \in U_{k(e, f)}$ olsun. O halde 
$M_{e}=\left\{x \in L: y \in I_{e}\right.$ olan bazı $y \in$

L ler için $y \| x$ dir. $\}$

ve

$M_{f}=\left\{x \in L: y \in I_{f}\right.$ olan bazl $y \in$

$L$ ler $i$ çin $y \| x$ dir. $\}$

olmak üzere

$K_{U^{2}}=\cup K_{S_{U_{1}^{2}}} \cup I_{e} \cup M_{e} \cup K_{T_{U_{2}^{2}}} \cup K_{S_{U_{2}^{2}}} \cup I_{f} \cup$

$M_{f} \cup I_{k}$

dir. $\square$

Ispat. $K_{T_{U_{1}^{2}}}, K_{S_{U_{1}^{2}}}, I_{e}, M_{e}, K_{T_{U_{2}^{2}}}, K_{S_{U_{2}^{2}}}, I_{f}, M_{f}$ ve $I_{k}$ kümeleri tanımlanışı gereği $K_{U^{2}}$ kümesinin alt kümeleridir. Buradan, $K_{T_{U_{1}^{2}}} \cup K_{S_{U_{1}^{2}}} \cup I_{e} \cup M_{e} \cup$ $K_{T_{U_{2}^{2}}} \cup K_{S_{U_{2}^{2}}} \cup I_{f} \cup M_{f} \cup I_{k} \subseteq K_{U^{2}}$ olduğu elde edilir. Tersine, $x \in K_{U^{2}}$ keyfi alalım. $\mathrm{O}$ halde, $\left(x<y\right.$ ve $\left.x \$_{U^{2}} y\right) \quad$ veya $\quad\left(y<x\right.$ ve $\left.y \$_{U^{2}} x\right)$ olacak şekilde $y \in L \backslash\{0,1\}$ mevcuttur. Keyfi bir $x \in L$ için $x \leq k, x \geq k$ veya $x \| k$ dir. İlaveten, $x \leq k$ ise $x \leq e, x \geq e$ veya $x \| e, x \geq k$ ise $x \leq f, x \geq f$ veya $x \| f$ olmak zorundadır. Yani bir $x \in L$ için mümkün durumlar $x \in[0, e]$, $x \in[e, k], x\|e, x \in[k, f], x \in[f, 1], x\| f$ veya $x \| k$ dir.

i) $\quad x \in[0, e]$ olsun.

i-1) $y \in[e, 1]$ olsun. Buradan, $\leq_{U^{2}}$ nin tanımı gereği $x \leq_{U^{2}} y$ olur. Bu ise, $x \in K_{U^{2}}$ olmasi ile çelişir.

i-2) $y \in[0, e]$ olsun. Bu durumda, $x<y, y<x$ veya $x \| y$ olabilir.

- $x<y$ olsun. Varsayalım ki, $x \leq_{T_{U_{1}^{2}}} y$ olsun. $\mathrm{O}$ halde, $U^{2}(l, y)=T_{U_{1}^{2}}(l, y)=x$ olacak şekilde $l \in[0, e]$ mevcuttur. Böylece, $x \leq_{U^{2}} y$ olur ki, bu bir çelişkidir. $\mathrm{O}$ halde, $x \$_{T_{U_{1}^{2}}} y$ olup $x \in K_{T_{U_{1}^{2}}}$ olur.

- $y<x$ olsun. Varsayalım ki, $y \leq_{T_{U_{1}^{2}}} x$ olsun. $\mathrm{O}$ halde, $U^{2}(l, x)=T_{U_{1}^{2}}(l, x)=y$ olacak şekilde $l \in[0, e]$ mevcuttur. Böylece, $y \leq_{U^{2}} x$ olur ki, bu bir çelişkidir. $\mathrm{O}$ halde, $y \mathbb{T}_{T_{1}^{2}} x$ olup $x \in K_{T_{U_{1}^{2}}}$ olur.

- $x \| y$ olsun. $x, y \in[0, e]$ olduğundan, $K_{T_{U_{1}^{2}}} \operatorname{nin}$ tanımından açıkça $x \in K_{T_{U_{1}^{2}}}$ olur. i-3) $y \| e$ olsun. $y, e$ ile kiyaslanamayan bir eleman olduğundan bu durum için $x \| y$ olmak zorundadır ( $x \leq y$ olursa $x \leq_{U^{2}} y$ olacağından, $y \leq x$ olursa $y \leq x \leq e$ olduğundan çelişki elde edilir). $y \| e$ ve $x \| y$ olduğundan $x \in M_{e}$ olur.

ii) $\quad x \in[e, k]$ olsun.

ii-1) $y \in[0, e](y \in[k, 1])$ olsun. Buradan, $\leq_{U^{2}}$ nin tanımı gereği $y \leq_{U^{2}} x\left(x \leq_{U^{2}} y\right)$ olur. Bu ise, $x \in K_{U^{2}}$ olması ile çelişir.

ii-2) $y \in[e, k]$ olsun. Bu durumda, $x<y, y<x$ veya $x \| y$ olabilir.

- $x<y$ olsun. Varsayalım ki, $x \leq_{S_{U_{1}^{2}}} y$ olsun. O halde, $U^{2}(l, x)=S_{U_{1}^{2}}(l, x)=y$ olacak şekilde $l \in[e, k]$ mevcuttur. Böylece, $x \leq_{U^{2}} y$ olur ki,

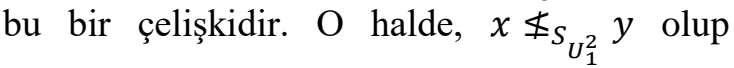
$x \in K_{S_{U_{1}^{2}}}$ olur.

- $y<x$ olsun. Varsayalım ki, $y \leq_{S_{U_{1}^{2}}} x$ olsun. $\mathrm{O}$ halde, $U^{2}(l, y)=S_{U_{1}^{2}}(l, y)=x$ olacak şekilde $l \in[e, k]$ mevcuttur. Böylece, $y \leq_{U^{2}} x$ olur ki,

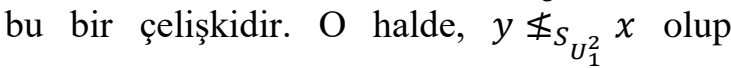
$x \in K_{S_{U_{1}^{2}}}$ olur.

- $x \| y$ olsun. $x, y \in[e, k]$ olduğundan, $K_{S_{U_{1}^{2}}} \operatorname{nin}$ tanımından açıkça $x \in K_{S_{U_{1}^{2}}}$ olur.

ii-3) $y \| e$ olsun. $y, e$ ile kıyaslanamayan bir eleman olduğundan bu durum için $x \| y$ olmak zorundadır ( $x \leq y$ olursa $e \leq x \leq y$ olduğundan, $y \leq x$ olursa $y \leq_{U^{2}} x$ olacağından çelişki elde edilir). $y \| e$ ve $x \| y$ olduğundan $x \in M_{e}$ olur.

iii) $\quad x \in[k, f]$ olsun.

iii-1) $y \in[0, k](y \in[f, 1])$ olsun. Buradan, $\leq_{U^{2}}$ nin tanımı gereği $y \leq_{U^{2}} x\left(x \leq_{U^{2}} y\right)$ olur. $\mathrm{Bu}$ ise, $x \in K_{U^{2}}$ olması ile çelişir.

iii-2) $y \in[k, f]$ olsun. Bu durumda, $x<y, y<x$ veya $x \| y$ olabilir.

$-x<y$ olsun. Varsayalım ki, $x \leq_{T_{U_{2}^{2}}} y$ olsun. $\mathrm{O}$ halde, $U^{2}(l, y)=T_{U_{2}^{2}}(l, y)=x$ olacak şekilde $l \in[k, f]$ mevcuttur. Böylece, $x \leq_{U^{2}} y$ olur ki, bu bir çelişkidir. $\mathrm{O}$ halde, $x \$_{T_{U_{2}^{2}}} y$ olup $x \in K_{T_{U_{2}^{2}}}$ olur.

- $y<x$ olsun. Varsayalım ki, $y \leq_{T_{U_{2}^{2}}} x$ olsun. $\mathrm{O}$ halde, $U^{2}(l, x)=T_{U_{2}^{2}}(l, x)=y$ olacak şekilde $l \in[k, f]$ mevcuttur. Böylece, $y \leq_{U^{2}} x$ olur ki, 
bu bir çelişkidir. $\mathrm{O}$ halde, $y \mathbb{T}_{T_{U_{2}^{2}}} x$ olup $x \in K_{T_{U_{2}^{2}}}$ olur.

- $x \| y$ olsun. $x, y \in[k, f]$ olduğundan, $K_{T_{U_{2}^{2}}} \operatorname{nin}$ tanımından açıkça $x \in K_{T_{U_{2}^{2}}}$ olur.

iii-3) $y \| f$ olsun. $y, f$ ile kıyaslanamayan bir eleman olduğundan bu durum için $x \| y$ olmak zorundadır $\left(x \leq y\right.$ olursa $x \leq_{U^{2}} y$ olacağından, $y \leq x$ olursa $y \leq x \leq f$ olduğundan çelişki elde edilir). $y \| f$ ve $x \| y$ olduğundan $x \in M_{f}$ olur.

iv) $\quad x \in[f, 1]$ olsun.

iv-1) $y \in[0, f]$ olsun. Buradan, $\leq_{U^{2}}$ nin tanımı gereği $y \leq_{U^{2}} x$ olur. Bu ise, $x \in K_{U^{2}}$ olmasi ile çelişir.

iv-2) $y \in[f$,1] olsun. Bu durumda, $x<y, y<x$ veya $x \| y$ olabilir.

- $x<y$ olsun. Varsayalım ki, $x \leq_{S_{U_{2}^{2}}} y$ olsun. $\mathrm{O}$ halde, $U^{2}(l, x)=S_{U_{2}^{2}}(l, x)=y$ olacak şekilde $l \in[k, 1]$ mevcuttur. Böylece, $x \leq_{U^{2}} y$ olur ki, bu bir çelişkidir. $\mathrm{O}$ halde, $x{\$_{S_{U_{2}^{2}}}} y$ olup $x \in K_{S_{U_{2}^{2}}}$ olur.

- $y<x$ olsun. Varsayalım ki, $y \leq_{S_{U_{2}^{2}}} x$ olsun. $\mathrm{O}$ halde, $U^{2}(l, y)=S_{U_{2}^{2}}(l, y)=x$ olacak şekilde $l \in[k, 1]$ mevcuttur. Böylece, $y \leq_{U^{2}} x$ olur ki, bu bir çelişkidir. $\mathrm{O}$ halde, $y \Varangle_{S_{U_{2}^{2}}} x$ olup $x \in K_{S_{U_{2}^{2}}}$ olur.

- $x \| y$ olsun. $x, y \in[e, k]$ olduğundan, $K_{S_{U_{2}^{2}}} \operatorname{nin}$ tanımından açıkça $x \in K_{S_{U_{2}^{2}}}$ olur.

iv-3) $y \| f$ olsun. $y, f$ ile kıyaslanamayan bir eleman olduğundan bu durum için $x \| y$ olmak zorundadır ( $x \leq y$ olursa $f \leq x \leq y$ olduğundan, $y \leq x$ olursa $y \leq_{U^{2}} x$ olacağından çelişki elde edilir). $y \| f$ ve $x \| y$ olduğundan $x \in M_{f}$ olur.

v) $\quad x \| e$ olsun. Bu takdirde, $x \in I_{e}$ dir.

vi) $\quad x \| f$ olsun. Bu takdirde, $x \in I_{f}$ dir.

vii) $\quad x \| k$ olsun. Bu takdirde, $x \in I_{k}$ dir.

Böylece, $K_{U^{2}} \subseteq K_{T_{U_{1}^{2}}} \cup K_{S_{U_{1}^{2}}} \cup I_{e} \cup M_{e} \cup K_{T_{U_{2}^{2}}} \cup$ $K_{S_{U_{2}^{2}}} \cup I_{f} \cup M_{f} \cup I_{k}$ olduğu elde edilir.

O halde, $K_{U^{2}}=K_{T_{U_{1}^{2}}} \cup K_{S_{U_{1}^{2}}} \cup I_{e} \cup M_{e} \cup K_{T_{U_{2}^{2}}} \cup$ $K_{S_{U_{2}^{2}}} \cup I_{f} \cup M_{f} \cup I_{k}$ dir.

2-uninormların uninormları kapsayan bir sinıf olduğu gerçeği göz önüne alındığında, 2uninormlar için (18) ile verilen $K_{U^{2}}=\cup K_{S_{U_{1}^{2}}} \cup$
$I_{e} \cup M_{e} \cup K_{T_{U_{2}^{2}}} \cup K_{S_{U_{2}^{2}}} \cup I_{f} \cup M_{f} \cup I_{k}$ eşitliğinin, (Kesicioğlu vd., 2017) çalışmasında uninormlar için elde edilen eşitliğin daha geneli olacağ açıktır. Dahası $U^{2}$ 2-uninormu için $e$ ve $f$ birim elemanları $e=f$ koşulunu sağlarsa $U^{2} \quad$ 2uninormu bir uninorm olur ve $U^{2}$ 2-uninormu için elde edilen (18) eşitliği (Kesicioğlu vd., 2017) de verilen eşitlikle çakışır. Bu çalışmada elde edilen eşitliğe dikkat edilirse, $I_{k}$ kümesinin varlığı da bu eşitliğin daha genel bir form olduğunu doğrular niteliktedir.

4.3. Sonuç $(L, \leq, 0,1)$ sınırlı bir kafes ve $U^{2} \in$ $U_{k(e, f)}$ olsun. $I_{e}=I_{f}=I_{k}=\emptyset$ ise

$K_{U^{2}}=K_{T_{U_{1}^{2}}} \cup K_{S_{U_{1}^{2}}} \cup K_{T_{U_{2}^{2}}} \cup K_{S_{U_{2}^{2}}}$

dir. $\square$

Ispat. $I_{e}=\varnothing$ ise $M_{e}=\emptyset$ ve $I_{f}=\emptyset$ ise $M_{f}=\varnothing$ olduğundan $I_{e}=I_{f}=I_{k}=\varnothing$ iken $K_{U^{2}}=K_{T_{U_{1}^{2}}} \mathrm{U}$ $K_{S_{U_{1}^{2}}} \cup K_{T_{U_{2}^{2}}} \cup K_{S_{U_{2}^{2}}}$ olduğu elde edilir.

4.4. Sonuç $(L, \leq, 0,1)$ sinırlı bir zincir ve $U^{2} \in U_{k(e, f)}$ olsun. Bu takdirde,

$K_{U^{2}}=K_{T_{U_{1}^{2}}} \cup K_{S_{U_{1}^{2}}} \cup K_{T_{U_{2}^{2}}} \cup K_{S_{U_{2}^{2}}}$

dir. $\square$

4.5. Önerme $(L, \leq, 0,1)$ sinırlı bir kafes ve $U^{2} \in U_{k(e, f)}$ olsun. $c \in L$ olmak üzere, $S_{U^{2}}$ siralamasına göre $c$ elemanı ile kiyaslanamayan elemanların kümesi

$I_{U^{2}}^{(c)}=\{x \in L \backslash\{0,1\}:$

$x, \leq_{U^{2}}$ sıralamasına göre c ile kıyaslanamazdır $\}(21)$

olmak üzere $K_{U^{2}}=\bigcup_{x \in L} I_{U^{2}}^{(x)}$ dir. $\square$

Ispat. $x \in L$ keyfi alındığında $I_{U^{2}}^{(x)}$ kümesinin tanımlanış1 gereği $I_{U^{2}}^{(x)} \subseteq K_{U^{2}}$ dir. Böylece, $\bigcup_{x \in L} I_{U^{2}}^{(x)} \subseteq K_{U^{2}}$ olduğu elde edilir.

Tersine, $y \in K_{U^{2}}$ keyfi alınsin. Böylece $\left[y<z\right.$ ve $\left.y \$_{U^{2}} z\right]$ veya $\left[z<y\right.$ ve $\left.z \$_{U^{2}} y\right]$ veya $z \| y$ olacak şekilde $z \in L \backslash\{0,1\}$ mevcuttur.

- $y<z$ ve $y \coprod_{U^{2}} z$ olsun. $z \leq_{U^{2}} y$ olduğunu varsayalım. Bu durumda, $z \leq y$ elde edilir ki bu $y<z$ ile çelişir. Böylece, $z \Varangle_{U^{2}} y$ olduğu elde edilir. $y \Varangle_{U^{2}} z$ ve $z \$_{U^{2}} y$ olduğundan, $y$, $\leq_{U^{2}}$ siralamasına göre $z$ ile kiyaslanamazdir. Böylece, $y \in I_{U^{2}}^{(z)}$ olur. 
- $z<y$ ve $z \$_{U^{2}} y$ olsun. $y \leq_{U^{2}} z$ olduğunu varsayalım. Bu durumda, $y \leq z$ elde edilir ki bu $z<y$ ile çelişir. Böylece, $y \sharp_{U^{2}} z$ olduğu elde edilir. $y \$_{U^{2}} z$ ve $z \$_{U^{2}} y$ olduğundan, $y$, $\leq_{U^{2}}$ sıralamasına göre $z$ ile kiyaslanamazdır. Böylece, $y \in I_{U^{2}}^{(z)}$ olur.

- $z \| y$ olsun. Buradan açikça, $y \sharp_{U^{2}} z$ ve $z \Varangle_{U^{2}} y$ olduğu elde edilir. Böylece, $y \in I_{U^{2}}^{(z)}$ olur.

O halde, $y \in K_{U^{2}}$ keyfi elemanı için, $y \in I_{U^{2}}^{(z)}$ olacak şekilde $z \in L \backslash\{0,1\}$ mevcuttur. $O$ halde, $y \in \cup_{x \in L} I_{U^{2}}^{(x)}$ olup $K_{U^{2}} \subseteq \bigcup_{x \in L} I_{U^{2}}^{(x)}$ olduğu elde edilir. $\square$

\section{Tartışma ve Sonuç}

$\mathrm{Bu}$ çalışmada şu sonuçlar elde edilmiştir. 2uninormlar üzerinde bir bağıntı tanımlanarak, bu bağıntının bir denklik bağıntısı olduğu elde edilmiștir. 2-uninormun belirlediği üçgensel normlar ve konormlardan elde edilen siralama ile 2-uninormlardan elde edilen suralama arasındanki ilişki araştırılmış ve aralarındaki ilişki ortaya konulmuştur. 2-uninormlardan elde edilen sıralama bağıntısına göre kıyaslanamayan tüm elemanların kümesi $K_{U^{2}}$ nin karakterize edilmiştir. $x$ elemanı ile kıyaslanamayan tüm elemanların kümesi $I_{U^{2}}^{(x)}$ ile $K_{U^{2}}$ ilişskisi ortaya konmuştur.

\section{Kaynaklar}

Akella, P., 2007. Structure of n-Uninorms, Fuzzy Sets and Systems, 158, 1631-1651.

Beliakov, G., Pradera, A. ve Calvo, T., 2007, Aggregation Functions: A Guide for Practitioners, in: Studies in Fuzziness and Soft Computing, 221, Springer, Berlin, Heidelberg, 361p.

Birkhoff G., 1967, Lattice Theory, 3 rd edition, Providence, Rhode Island, 418p.

Ertuğrul, Ü., 2017a. Some properties of orders generated by uninorm and 2-uninorm, New Trends in Mathematical Sciences, 1, 278286.

Ertuğrul, Ü., 2017b. A Way to Obtain 2-Uninorm on Bounded Lattice from Uninorms Defined on Subintervals of Bounded Lattice, New Trends in Mathematical Sciences, 2, 1-9.
Ertuğrul, Ü., 2018. Construction of nullnorms on bounded lattices and an equivalence relation on nullnorms, Fuzzy Sets and Systems, https://doi.org/10.1016/j.fss.2017.07.020.

Ertuğrul, Ü., Kesicioğlu, M.N. ve Karaçal, F., 2016. Ordering Based on Uninorms, Information Sciences, 330, 315-327.

Ertuğrul, Ü., Kesicioğlu, M.N. ve Karaçal, F., 2017. Ordering Based on 2-Uninorms on Bounded Lattices, New Trends in Mathematical Sciences, 1, 287-293.

Grabish, M., Marichal, J.-L., Mesiar, R. ve Pap, E., 2009, Aggregation Functions, Cambridge University Press, 460p.

Hliněná, D., Kalina, M. ve Král P., 2014. Preorders and Orders Generated by Conjunctive Uninorms, Inf. Proc. Manage. Uncert. Knowl. Based Syst., 30, 807-817.

Karaçal, F., Ertuğrul, Ü. ve Mesiar, R., 2017. Characterization of Uninorms on Bounded Lattices, Fuzzy Sets and Systems, 308, 5471.

Karaçal, F. ve Kesicioğlu, M.N., 2011. A T-partial Order Obtained From T-norms, Kybernetika, 47, 300-314.

Karaçal, F. ve Mesiar, R., 2015. Uninorms on Bounded Lattices, Fuzzy Sets and Systems, 261, 33-43.

Kesicioğlu, M.N., Ertuğrul, Ü. ve Karaçal, F., 2017. An Equivalence Relation Based On The U-Partial Order, Information Sciences, 411, 39-51.

Kesicioğlu, M.N., Karaçal, F. ve Mesiar, R., 2015. Order-equivalent Triangular Norms, Fuzzy Sets and Systems, 268, 59-71.

Kesicioğlu, M.N. ve Mesiar, R., 2014. Ordering Based on Implications, Information Sciences, 276, 377-386.

Yager, R.R. ve Rybalov, A., 1996. Uninorm Aggregation Operators, Fuzzy Sets and Systems, 80, 111-120. 